\title{
DO THE PRESSURE CHANGES OF NEONATAL NON-SYNCHRONISED NIPPV (NS NASAL INTERMITTENT POSITIVE PRESSURE VENTILATION) CONFER ADVANTAGES OVER CPAP, OR ARE HIGH CPAP PRESSURES AS EFFECTIVE?
}

\author{
L.S. Owen ${ }^{1,2,3,4}$, C.J. Morley ${ }^{1,2,3}$, P.G. Davis ${ }^{1,2,3}$ \\ ${ }^{1}$ Newborn Research, Royal Womens Hospital, ${ }^{2}$ Murdoch Childrens Research Institute, ${ }^{3}$ Dept. of Obstetrics \\ and Gynaecology, University of Melbourne, Melbourne, VIC, Australia, ${ }^{4}$ Faculty of Medical Sciences, \\ University of Bristol, Bristol, UK
}

Background: Neonatal NIPPV is widely used. However, its mechanisms of action are not clear, especially when NIPPV is not synchronised with spontaneous breathing.

Aim: To examine whether nsNIPPV has benefits over CPAP, when CPAP is delivered at either the average pressure generated during NIPPV (high level CPAP: HiCPAP), or at the PEEP delivered during NIPPV (standard CPAP: StCPAP).

Method: We studied 10 infants receiving ventilator-generated nsNIPPV (median gestation $25^{+4}$ weeks, birth weight 784g, postnatal age 31 days). Infants received 30 minutes each of nsNIPPV, HiCPAP and StCPAP, in random order. Delivered pressures, oxygen saturation, transcutaneous oxygen and carbon dioxide levels, heart and respiratory rates, inspired oxygen and tidal volumes were recorded using Spectra software.

Results: Median settings were 20/7 $\mathrm{cm} \mathrm{H}_{2} \mathrm{O}$, rate 20/min during nsNIPPV, $7 \mathrm{~cm} \mathrm{H}_{2} \mathrm{O}$ during StCPAP and $8.5 \mathrm{~cm} \mathrm{H}_{2} \mathrm{O}$ during HiCPAP. There were no clinical or statistical differences between the modes in terms of inspired or measured levels of oxygen or carbon dioxide, respiratory or heart rates, bradycardias or tidal volumes.

More apnoeas and desaturations were seen during $\operatorname{StCPAP}(\mathrm{p}=0.01$ and $\mathrm{p}=0.03)$. Infants spent less time with saturations below $80 \%$ on HiCPAP than nsNIPPV ( $4.2 \%$ vs. $10.1 \%$ respectively), whereas there were fewer, shorter apnoeas on nsNIPPV than HiCPAP.

Conclusions: There were minimal differences between ventilator-generated nsNIPPV and HiCPAP. This suggests that any advantage of nsNIPPV may be due to higher overall pressures, rather than the NIPPV pressure variations themselves. In this short-term cross-over study higher CPAP pressures appeared safe and just as effective as nsNIPPV. 